\title{
Active-reactive scheduling of active distribution system considering interactive load and battery storage
}

\author{
Qixin Chen, Xiangyu Zhao and Dahua Gan * (D)
}

\begin{abstract}
Distributed generation (DG) are critical components for active distribution system (ADS). However, this may be a serious impact on power system due to their volatility. To this problem, interactive load and battery storage may be a best solution. This paper firstly investigates operation characteristics of interactive load and battery storage, including operation flexibility, inter-temporal operation relations and active-reactive power relations. Then, a multi-period coordinated activereactive scheduling model considering interactive load and battery storage is proposed in order to minimize overall operation costs over a specific duration of time. The model takes into accounts operation characteristics of interactive load and battery storage and focuses on coordination between DGs and them. Finally, validity and effectiveness of the proposed model are demonstrated based on case study of a medium-voltage 135-bus distribution system.
\end{abstract}

Keywords: Active distribution system, Active-reactive scheduling, Interactive load, Battery storage

\section{Introduction}

Active distribution system (ADS) is defined as distribution networks that have systems in place to control a combination of distributed energy resources (DERs), including distributed generators (DGs), battery storage, demand response, etc. [1]. In recent, high levels of DERs that could be efficiently scheduled are being integrated in order to achieve specific operational objectives, for example, costs minimization. Therefore, it would be necessary for the Distribution System Operators (DSOs) to transform from the traditional "passive" unidirectional flow operation approach to novel "active" bi-directional flow operation approach [2]. To this end, a critical challenge is to formulate the operation characteristics of different kinds of DERs and integrate them into the scheduling scheme of ADS.

At present, many interesting researches related to the operation of ADS have been conducted [3-6]. Pilo et al. [1] and Keane et al. [7] proposed models and methodology to minimize system operation cost by optimizing the production of the local DGs, including the wind turbine, photovoltaic, considering power exchanges with

\footnotetext{
* Correspondence: gandahua0151@sina.com

State Key Lab of Power Systems; Dept. of Electrical Engineering, Tsinghua University, Beijing 100084, China
}

the main distribution system. As power outputs of DGs are always restricted by meteorological factors [7], the volatility characteristics become a heavy burden to the DSOs. In this case, battery storage could serve as an option for accommodating volatile outputs of DGs [8]. An optimal model for ADS proposed in [9] contains DGs and battery storage, but only take the capacity limitation of battery storage into consideration. Further, the relation between active-reactive power outputs of battery storage is considered in [10-12], and the active-reactive coordination model for DGs and battery storage is proposed.

Though battery storage could solve the volatility of DGs, their high investment cost may increase the total operation cost of the distribution system [13]. Therefore, demand response may be another solution. In fact, demand response is a price mechanism between DSOs and the local users, and interactive load is an important type of demand response. Under the agreement, the DSOs could change the original load shape, while users could get some payback from the DSOs. Compared to battery storage, demand response could achieve similar aims and, at the same time, there would be hardly no investment cost. Dozens of demand response projects have been established and operated in many countries 
[14-18]. While an optimal model of ADS considering demand response and battery storage is not yet proposed.

Based on the above analysis, this paper focuses on multi-period coordinated active-reactive scheduling of ADS considering demand response and battery storage. Firstly, we design a new form of demand response, namely interactive load and the structure of battery storage is also analyzed. Then, the problem description and the mathematical model of interactive load and battery storage are presented. Based on these model, a novel multi-period active-reactive coordinated scheduling model is proposed for integrated operation of ADS, in order to minimize overall operation costs over a specific duration of time. The model takes into accounts operation characteristics of various DERs and formulates multi-period operation of ADS. Finally, validity and effectiveness of the proposed model are demonstrated based on case study of a medium-voltage 135-bus distribution system.

\section{Flexible operation of interactive load and battery storage}

\subsection{Introduction to interactive load}

According to the report of Federal Energy Regulatory Commission about demand response (DR) and advanced metering, DRs could be divided into 15 types on the basis of their response form, such as Direct Load Control, Interruptible Load, Critical Peak Pricing with Control, etc.

Interactive Load designed in this paper is a combination of Direct Load Control, Demand Bidding and Buyback. Its basic feature is that the DSOs would obtain the right to invoke the electrical equipment according to the agreement, thus the shape of power load could be changed to the most economical way. While at the same time, consumers could get economic compensation due to their participations in load shifting.

According to the effect on the load curve, interactive load could be divided into Peak Cutting Load and Peak Shifting Load. As shown Fig. 1, Peaking Cutting Load

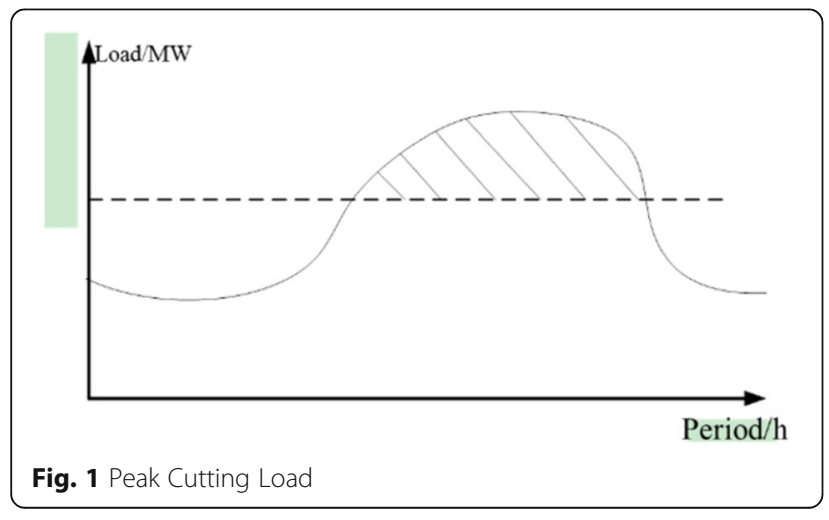

would restrict power load during Peaking period and cause the loss of electricity consumption. While the load cut during peak period by Peak Shifting Load would be shifted to the valley period and the electricity consumption could be remained, as shown as Fig. 2. (Please delete the following 'Fig. 2 Peak Shifting Load', it's added automatically when building up the PDF and I cannot tell why).

In fact, Peak Cutting Load is another category of Peak Shifting Load whose load cut during peak period wouldn't be compensated. And considering that Peak Cutting Load may cause uncontrollable load rebound during valley period, this paper focuses on the Shifting one. Furthermore, it could be divided into two types, namely Shapeable Load and Removable Load. Their characteristic and modeling will be presented in the following chapters.

\subsection{Characteristic and modeling of shapeable load 2.2.1 Introduction to shapeable load}

As shown in Fig. 3, Shapeable Load could change the load shape during load shifting according to the agreement, but electricity consumption and the duration time of Shapeable load should be remained.

\subsubsection{Load shifting potential analysis of shapeable load}

Thermal storage, such as large-scale central conditioning system, is an important resource of Demand Side Response. Its application could be described as Shapeable Load and its electrical characteristics is described in.

The thermal storage projects implemented in China are investigated in this paper and the load shifting potential is listed in Table 1.

\subsection{Characteristic and modeling of removable load 2.3.1 Introduction to removable load}

Compared with Shapeable Load, Removable Load has strict requirements when shifting. As shown in Fig. 4, it requires that load shape should stay the same.

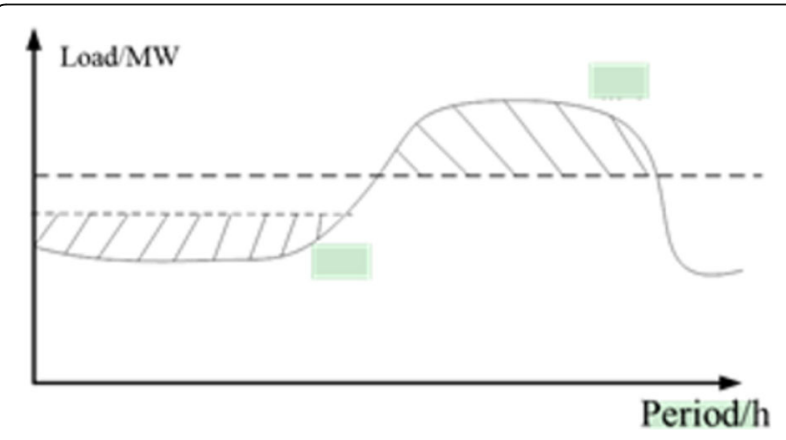

Fig. 2 Peak Shifting Load 


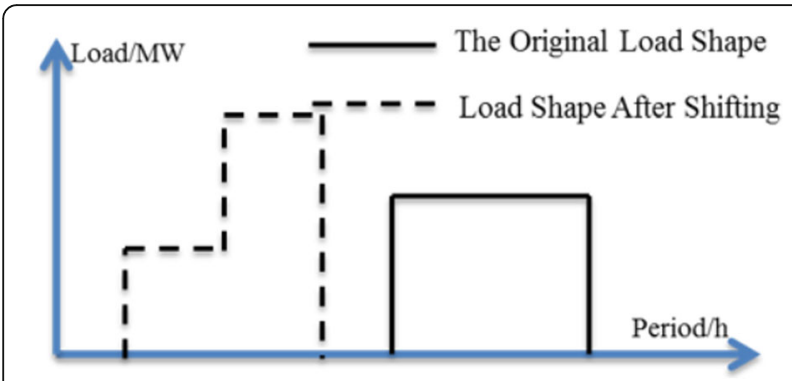

Fig. 3 Shapeable Load

\subsubsection{Load shifting potential analysis of removable load}

Removable Load can be used to describe the production process transfer of industrial user. Industrial production tend to have a relatively fixed production process. So when transferring their production process, their load shape should be the same.

This paper investigates load shifting potential of industrial users in Beijing, China. The results of the survey are shown in Table 2. Total load shifting potential is $90 \sim 120 \mathrm{MW}$, about $2.5 \%$ of the peak load. If all the load shifting potential of industrial users could be made full use of, power grid operation will be improved significantly.

\subsection{Characteristic and modeling of battery storage}

According to the energy storage form, battery storage can be divided into superconducting energy storage, chemical battery energy storage, flywheel energy storage, etc. Though their energy storage form are quite different, they share similar structure. As shown in Fig. 5, generally speaking, battery storage includes two components. Storage unit is used for energy storage, its capacity decides how much power energy can be stored in

Table 1 Load shifting potential analysis of thermal storage in

China

\begin{tabular}{|c|c|c|}
\hline Location & Project Name & Load Shifting Potential \\
\hline Beijing & $\begin{array}{l}94 \text { Thermal Storage } \\
\text { projects in } 2002\end{array}$ & $200 \mathrm{MW}$ \\
\hline Beijing & Industrial consumer & $\begin{array}{l}100 \mathrm{MW}(1996), 2.3 \% \text { of } \\
\text { the peak load }\end{array}$ \\
\hline Guangzhou & $\begin{array}{l}\text { Cold storage project at } \\
\text { Economic Trade } \\
\text { Commission building } \\
\text { in } 2006\end{array}$ & $240 \mathrm{~kW}$ \\
\hline Guangxi Province & $\begin{array}{l}\text { Thermal Equipment in } \\
118 \text { consumers }\end{array}$ & $\begin{array}{l}162 \mathrm{MW}, 2.3 \% \text { of the } \\
\text { peak load }\end{array}$ \\
\hline Shanghai & $\begin{array}{l}\text { Cold storage project } \\
\text { in Jindu Building }\end{array}$ & $220 \mathrm{~kW}$ \\
\hline Shanghai & $\begin{array}{l}\text { Central Air conditions } \\
\text { system in hotel and mall }\end{array}$ & $598 \mathrm{MW}$ \\
\hline
\end{tabular}

As a conclusion, load shifting potential of Shapeable Load would be enormous as thermal storage projects are promoted widely in China

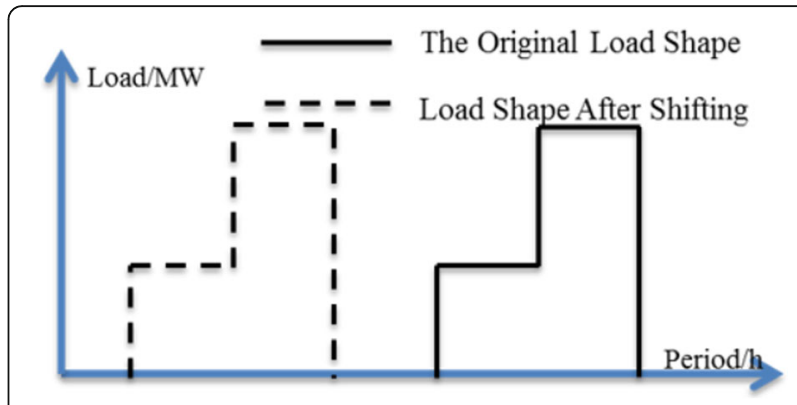

Fig. 4 Removable Load

the battery. Power conditioning system (PCS) is an electric power device, which is used to exchange power energy with the power grid. Its control mode decides active and reactive power of the battery.

\section{Methods}

\subsection{Modeling of interactive load}

This section is focus on formulations on operation characteristics of shapeable load and the removable one. The analyzed characteristics include load shifting cost curve, load shifting position and constraints for load shape and electricity consumption.

\subsubsection{Load shifting cost curve}

For describing the load shifting cost, load shifting cost curve is designed as shown in Fig. 6. The farther load is shifted, the heavier it changes the habit of users, so the cost will be more expensive. Thus, we can get a load shifting cost curve like a "tub". The load shifting cost could be expressed as:

$$
\begin{aligned}
C^{I L}= & \sum_{i=1}^{T} \sum_{j=1}^{N S L} \lambda_{i, j}^{\text {shift }} \times P_{i, j}^{S L, A}+\sum_{i=1}^{T} \sum_{j=1}^{N R L} \lambda_{i, j}^{\text {shift }} \\
& \times P_{i, j}^{R L, A}
\end{aligned}
$$

where $C^{I L}$ denotes total load shifting cost of interactive load, including two parts, the cost of shapeable load and it of removable load. $T$ devotes the total period number. NSL

Table 2 Load shifting potential analysis of industrial users in Beijing

\begin{tabular}{ll}
\hline Industrial User Name & Load Shifting Potential(MW) \\
\hline Shougang Corporation & 50 \\
Tegang Corporation & $10 \sim 20$ \\
Yanhua Corporation & $5 \sim 10$ \\
Chemical Industry & $10 \sim 20$ \\
Building Materials Industry & 10 \\
Total & $90 \sim 120$ \\
\hline
\end{tabular}




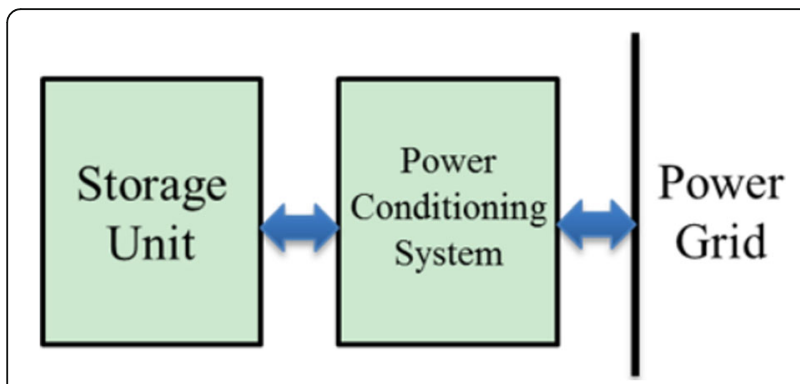

Fig. 5 The Structure of Battery Storage (should be Fig. 5, it's changed to Fig. 1 after building the PDF and I cannot figure out why)

and NRL respectively denote the number of shapeable load and removable load. $\lambda_{i, j}^{\text {shift }}$ denotes the load shift cost of shapeable load or removable load $j$ at period $i . P_{i, j}^{S L, A}$ and $P_{i, j}^{R L, A}$ respectively denote the load of shapeable load $j$ and removable load $j$ at period $i$ after their shifting.

\subsubsection{Load shifting position}

In order to describe load shifting position of interactive load, we introduce a set of state variables, $\eta_{i, j}^{S L}$ and $\eta_{i, j}^{R L}$, respectively denoting whether the head of shapeable load $j$ and removable load $j$ is shifted to period $i$. It should be ensured that every head of shapeable load or removable load can be shifted to only one position. And in order to not affect operation of next day, the state variables from $\eta_{T-T_{0}, j}^{S L}$ to $\eta_{T, j}^{S L}$ and from $\eta_{T-T_{0}, j}^{R L}$ to $\eta_{T, j}^{R L}$ are set to 0 . It could be expressed as:

$$
\left\{\begin{array}{l}
\sum_{i=1}^{T} \eta_{i, j}^{S L}=1 \\
\sum_{i=1}^{T} \eta_{i, j}^{R L}=1 \\
\eta_{i, j}^{S L}=0 \quad i=T-T_{0, j}^{S L}+1, \cdots T \\
\eta_{i, j}^{R L}=0 \quad i=T-T_{0, j}^{R L}+1, \cdots T
\end{array}\right.
$$

Where $T_{0, j}^{S L}$ and $\eta_{0, j}^{R L}$ respectively denotes the length of shapeable load $j$ and removable load $j$.
3.1.3 Constraints for load shape and electricity consumption Load shape of shapeable load could be changed before and after shifting, but electricity consumption should be the same. While load shape of removable load should be the same. Load at period $i$ could be expresses as:

$$
\left\{\begin{array}{l}
\sum_{m=1}^{T} P_{m, j}^{S L, A}=\sum_{m=1}^{T_{0, j}^{S L}} P_{m, j}^{S L, B} \\
\underline{P}_{j}^{S L} \times \sum_{m=i-T_{0, j}^{S L}+1}^{i} \eta_{m, j}^{S L} \leq P_{i, j}^{S L, A} \leq \bar{P}_{j}^{S L} \times \sum_{m=i-T_{0, j}^{S L}+1}^{i} \eta_{m, j}^{S L} \\
P_{i, j}^{R L, A}=\sum_{m=1}^{T_{0, j}^{R L}} \eta_{i-m+1, j}^{S L} \times P_{m, j}^{R L, B}
\end{array}\right.
$$

where $P_{i, j}^{S L, A}$ and $P_{i, j}^{R L, A}$ respectively denote the load of shapeable load $j$ and removable load $j$ at period $i$ before their shifting. $\underline{P} j^{S L}$ and $\bar{P}_{j}^{S L}$ denote the upper and lower bounds of shapeable load $j$.

The relation between active and reactive load of interactive load is complicated. It's assumed that their power factor stay the same before and after shifting. It could expressed as:

$$
\left\{\begin{array}{c}
P_{i, j}^{S L, A}=C_{j}^{S L} \times Q_{i, j}^{S L, A} \\
P_{i, j}^{S L, B}=C_{j}^{S L} \times Q_{i, j}^{S L, B} \\
P_{i, j}^{R L, A}=C_{j}^{R L} \times Q_{i, j}^{R L, A} \\
P_{i, j}^{R L, B}=C_{j}^{R L} \times Q_{i, j}^{R L, B}
\end{array}\right.
$$

where $C_{j}^{S L}$ and $C_{j}^{R L}$ respectively denote the fixed coefficient between active load and reactive load of shapeable load $j$ and removable load $j$.

\subsection{Modeling of battery storage}

This section is focus on formulations on operation characteristics of battery storage. The analyzed

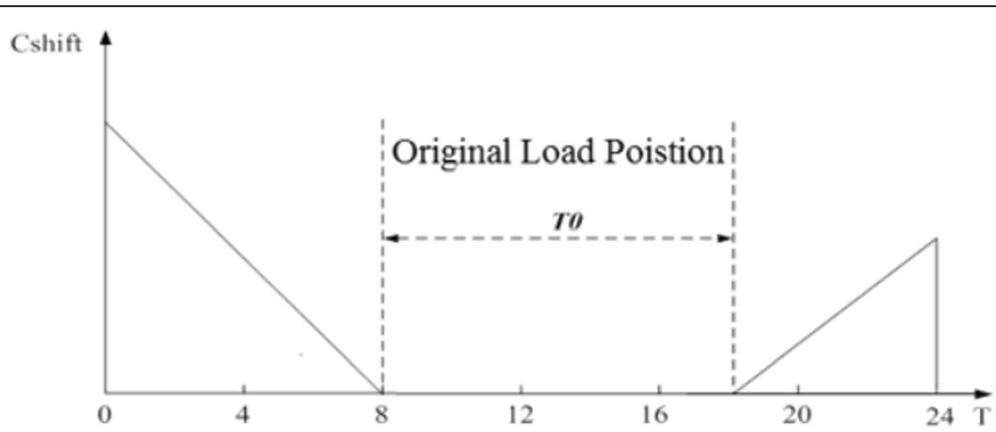

Fig. 6 Load Shifting Cost Curve (should be Fig. 6, it's changed to Fig. 2 after building the PDF and I cannot figure out why) 
characteristics include operation cost for battery, constraints for energy storage, power exchange and state transition number.

\subsubsection{Constraints for energy storage}

It must be insured that the stored energy is within the storage capacity limitation at any period. Besides power loss should also be taken into consideration, including the loss within charging process, discharging process and storage process. This paper converts all these losses into charging process in order to simplify the issue. Then the stored energy at final period should be equal to that at initial period after considering limitations. All these constraints could be expressed as:

$$
\left\{\begin{array}{l}
\underline{E}_{j}^{S} \leq E_{j}^{S, p r e}+\sum_{n=1}^{i}\left(\mu_{j}^{S} \times P_{n, j}^{S, C} \times \Delta T-P_{n, j}^{S, D} \times \Delta T\right) \leq \bar{E}_{j}^{S} \\
i=1 \cdots T \\
\sum_{n=1}^{T} \mu_{j}^{S} \times P_{n, j}^{S, C}-P_{n, j}^{S, D}=0
\end{array}\right.
$$

where $\underline{E} j^{S}$ and $\bar{E}_{j}^{S}$ denote the upper and lower bounds of storage capacity of battery storage $j . E_{j}^{S, \text { pre }}$ denotes the initial stored energy. $P_{i, j}^{S, C}$ and $P_{i, j}^{S, D}$ respectively denote the active power charged or discharged between battery storage $j$ and the grid at period $i . u_{j}^{S}$ is the loss rate of the storage battery $j$ at charge process.

\subsubsection{Constraints for power exchange}

Device type and control mode of PCS decides the active and reactive power characteristics of battery storage. Nowadays, full-controlled electrical devices are widely used in battery storage, thus making the battery could operating in four-quadrant zone as shown in Fig. 7. Constraints for active and reactive power could be expressed as:

$$
\left\{\begin{array}{l}
\left(P_{i, j}^{S, C}\right)^{2}+\left(Q_{i, j}^{S}\right)^{2} \leq\left(S_{j}^{S}\right)^{2} \\
\left(P_{i, j}^{S, D}\right)^{2}+\left(Q_{i, j}^{S}\right)^{2} \leq\left(S_{j}^{S}\right)^{2} \\
P_{i, j}^{S, C} \geq 0 \\
P_{i, j}^{S, D} \geq 0
\end{array}\right.
$$

where $Q_{i, j}^{S}$ denote reactive power of the battery storage $j$ at period $i$ and $S_{j}^{S}$ denote the power bound to the battery storage $j$.

\subsubsection{Constraints for state transition number}

Lifetime of battery storage is highly affected by their state transition number. To prolong the lifetime of battery storage, only one cycle charge/discharge per day is

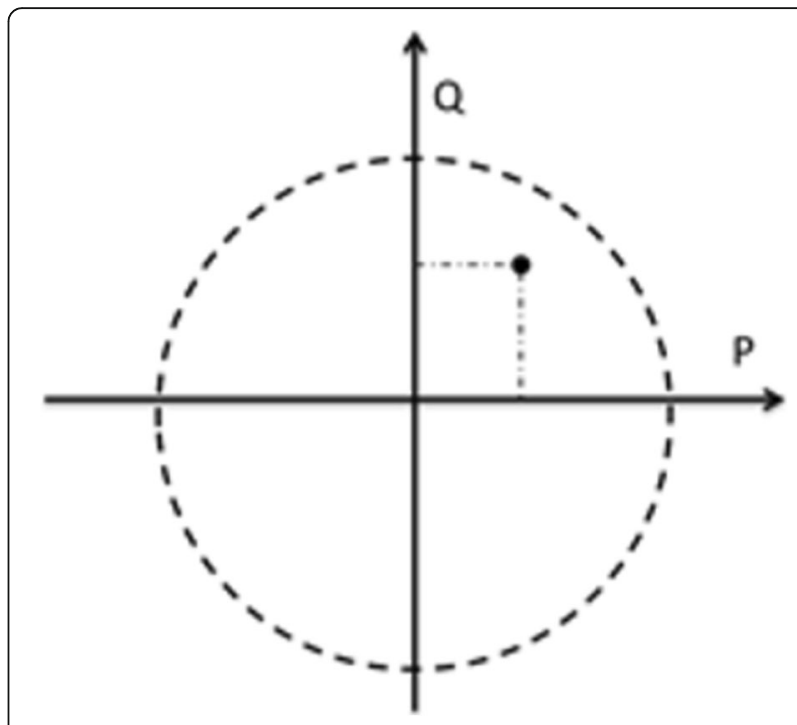

Fig. 7 Active and Reactive Power of Battery Storage

typically chosen for optimal operation. Considering the continuity of battery storage operation, as shown in Fig. 8, every storage would go through one charge/discharge state change and one discharge-charge state change. These constraints could be expressed as:

$$
\left\{\begin{array}{l}
\eta_{i, j}^{S, S}, \eta_{i, j}^{S, C D}, \eta_{i, j}^{S, D C} \in\{0,1\} \\
\eta_{i, j}^{S, S}-\eta_{i-1, j}^{S, S}-\eta_{i, j}^{S, C D}+\eta_{i, j}^{S, D C}=0 \\
\sum_{i=1}^{T} \eta_{i, j}^{S, C D}=1 \\
\sum_{i=1}^{T} \eta_{i, j}^{S, D C}=1
\end{array}\right.
$$

where $\eta_{i, j}^{S, S}$ denotes the state of battery storage $j$ at period $i$. It will be set to one when the battery is in discharge process, while zeros corresponds to the charge process. $\eta_{i, j}^{S, C D}$ and $\eta_{i, j}^{S, D C}$ respectively denote the operation state change from charge process to discharge process and from discharge process to charge process. It's guaranteed by the second sub-formula in (8) that $\eta_{i, j}^{S, C D}$ will be assigned as one if the operation state of battery storage $j$ is changed from charge process to discharge process at period $i$. While it will be zero at other period. Similar situation can be implemented to $\eta_{i, j}^{S, D C}$ as it will be assigned as one if the operation state of battery storage $j$ is changed from discharge process to charge process at period $i$. Besides the third and fourth sub-formula guarantee that the state transition number would be just one. 


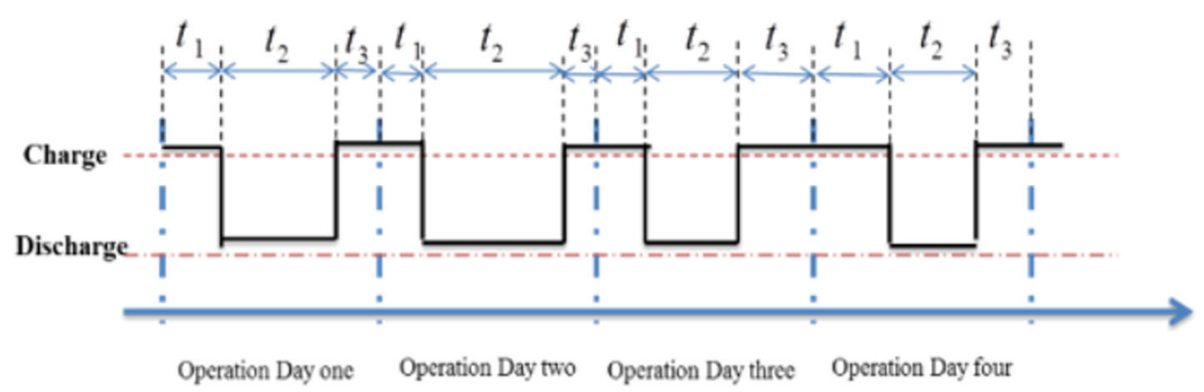

Fig. 8 The Continuity of battery storage

\subsubsection{Operation cost for battery storage}

Operation cost for battery storage includes the depreciation of investment cost and daily operation cost. The depreciation of investment cost could be expressed by a fixed constant and daily operation cost could be calculated by the exchanged power. These relation could be expressed as:

$$
C_{j}^{B S}=M_{j B S, D}+\sum_{i=1}^{T}\left(\lambda_{j}^{S, P} P_{i, j}^{S, C}+\lambda_{j}^{S, Q} Q_{i, j}^{S}\right)
$$

where $C_{j}^{B S}$ and $M_{j B S, D}$ denote the total operation cost and the depreciation of investment cost of battery storage $j$. $\lambda_{j, s}^{s, p}$ and $\lambda_{j, s}$ are the active and reactive cost coefficients of battery storage $j$.

\section{Multi-period coordinated scheduling model considering battery storage and interactive load}

\subsection{Decision variables}

The decision variables include continuous ones for active and reactive power of source bus, distributed generation, battery storage as well as interactive load, and "0-1" binary integer ones for operation states of battery storage and interactive load. Noted that there are two operation processes for battery storage, discharge and charge, three sets of decision variables would be assigned for each process.

\subsubsection{Continues decision variables}

Continues decision variables include $P_{i}^{\text {Sou }}, Q_{i}^{\text {Sou }}, P_{i, j}^{D G}$, $Q_{i, j}^{D G}, P_{i, j}^{S L, A}, Q_{i, j}^{S L, A}, P_{i, j}^{R L, A}, Q_{i, j}^{R L, A}, P_{i, j}^{S, C}, P_{i, j}^{S, D}, Q_{i, j}^{S}, V_{i, j}, \theta_{i, j}$, where $P_{i}^{\text {Sou }}$ and $Q_{i}^{\text {Sou }}$ denote the active and reactive power of source bus at time interval $i$ and $P_{i, j}^{D G}$ and $Q_{i, j}^{D G}$ are the active and reactive power of distributed generation $j$ at time interval $i$. $V_{i, j}$ and $\theta_{i, j}$ denote the voltage amplitude and angle of bus $j$ at time interval $i$.

\subsection{2 "0-1" binary integer decision variables}

“0-1" binary integer decision variables include $\eta_{i, j}^{S L}, \eta_{i, j}^{S, S}$, $\eta_{i, j}^{C D}, \eta_{i, j}^{D C}$

\subsection{Objective function}

The object is to minimize the overall operation costs of distribution system over a specific duration of time, including power generation/operation costs from source bus, distribution generation, and battery storage, load shifting cost of interactive load and investment costs of battery storage if it's built by DSO. It can be expressed as:

$$
\min \left\{\begin{array}{l}
\sum_{i=1}^{T}\left(\lambda_{i}^{\text {Sou, } P} \times P_{i}^{\text {Sou }}+\lambda_{i}^{\text {Sou }, Q} \times Q_{i}^{\text {Sou }}\right) \\
+\sum_{i=1}^{T} \sum_{j=1}^{N G}\left(\lambda_{j}^{D G, P} P_{i, j}^{D G}+\lambda_{j}^{D G, Q} Q_{i, j}^{D G}\right) \\
+\sum_{i=1}^{T} \sum_{j=1}^{N S}\left(\lambda_{j}^{S, P} P_{i, j}^{S, C}+\lambda_{j}^{S, Q} Q_{i, j}^{S}\right)+\sum_{j=1}^{N S} M_{j}^{B S, D} \\
+\sum_{i=1}^{T} \sum_{j=1}^{N S L} \lambda_{i, j}^{\text {shift }} \times P_{i, j}^{S L, A}+\sum_{i=1}^{T} \sum_{j=1}^{N R L} C_{i, j}^{\text {shift }} \times P_{i, j}^{R L, A}
\end{array}\right\}
$$

Where $\lambda$ denotes cost coefficients. The superscripts of the variables and parameters in (10) are used to distinguish different kinds of DERs (Sou, DG and $S, P$ ) and active and reactive power output $(P, Q) . N G$ and $N S$ respectively denote sets of distribution generation and battery storage.

In (9), generation costs of the source bus are related to its active and reactive power. The costs of reactive power might come from contracts or auxiliary markets, these two mechanisms could both be reflected by cost coefficients. The same situation would be implemented to distributed generations.

\subsection{Constraints}

Constraints mainly consist of two categories, respectively related to system operation and various DERs.

\subsubsection{System operation constraints}

System operation constraints are safety operation constraints for the distribution system, including constraints 
for power balance, bus voltage and transmission power flow.

Power balance constraint on source bus is expressed as:

$$
\left\{\begin{array}{l}
P_{i}^{\text {Sou }}-P_{i, s}^{\text {load }}=F_{1}\left(V_{i}, \theta_{i}\right) \\
Q_{i}^{\text {Sou }}-Q_{i, s}^{\text {load }}=F_{2}\left(V_{i}, \theta_{i}\right)
\end{array}\right.
$$

Power balance constraints on other buses are expressed as:

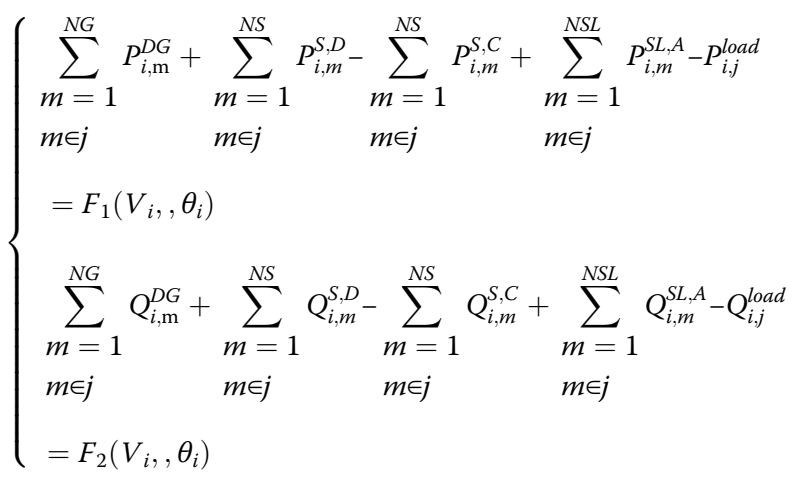

where $P_{i, j}^{\text {load }}$ and $Q_{i, j}^{\text {load }}$ respectively denote active and reactive load of bus $j$ at time interval $i . V_{i}$ is vector of bus voltage magnitude and $\theta_{i}$ is vector of bus voltage angle at time interval i. $F_{1}$ and $F_{2}$ are active and reactive power flow functions.

Note that circuit parameters and operation state of distribution system are quite different from those of transmission system, transmission power flow should be calculated based on AC power flow.

Constraints on transmission power flow are expressed as:

$$
\left(\underline{S}^{\text {line }}\right)^{2} \leq\left(P_{i, j}^{\text {line }}\right)^{2}+\left(Q_{i, j}^{\text {line }}\right)^{2} \leq\left(\bar{S}_{j}^{\text {line }}\right)^{2}
$$

Where $\bar{S}_{j}^{\text {line }}$ and $\underline{S} j^{\text {line }}$ are upper and lower capacity limitations of line $j . P_{i, j}^{\text {line }}$ and $Q_{i, j}^{\text {line }}$ are the active and reactive transmission power flow of line $j$ at time interval $i$.

Constraints on bus voltage are expressed as:

$$
\left\{\begin{array}{l}
\underline{V}^{b} \leq V_{i, j} \leq \bar{V}^{b} \\
V_{i, j}=V^{s, s e t} \\
\theta_{i, j}=0
\end{array}\right.
$$

where $V_{i, s}$ and $\theta_{i, s}$ denote voltage magnitude and angle of source bus. $\bar{V}_{j}^{b}$ and $\underline{V} j^{b}$ respectively denote upper and lower limitation of voltage magnitude of bus $j$.

\subsubsection{DER operation constraints}

Operation constraint constraints on distributed generation, battery storage and interactive load are established based on their operation characteristics.

The generation range and the relations on activereactive power output are two important operation characteristics for distributed generation. The output range of wind turbine and photovoltaic is based on their power prediction, while power output of gas turbine should be adjusted within its generation capacity limitation. Distributed generation are always connected to distributed system through power electronics equipment. Relations on active -reactive power output are mainly decided by types and control strategies of the equipment. And there are mainly two kinds of control strategies: constant voltage control (CVC) and constant power factor control (CPFC). These relations could be formulated as:

$$
\left\{\begin{array}{l}
\underline{P}_{i, j}^{G} \leq P_{i, j}^{G} \leq \bar{P}_{i, j}^{G} \\
\underline{Q}_{i, j}^{G} \leq Q_{i, j}^{G} \leq \bar{Q}_{i, j}^{G} \\
C P F C: \quad P_{i, j}^{G}=C_{j}^{G} \times Q_{i, j}{ }^{G} \\
C V C: \quad V_{i, j}^{G}=V_{j}^{G, s e t}
\end{array}\right.
$$

where $P_{i, j}^{G}$ and $Q_{i, j}^{G}$ respectively denote the active and reactive power output of distributed generation $j$ at period $i$ and $\bar{P}_{i, j}^{G}, \underline{P} i, j^{G}, \bar{Q}_{i, j}^{G}$ and $Q i, j^{G}$ are their upper and lower bounds. $C_{j}^{G}$ is the controlled power factor between active and reactive power of distributed generation $j . V_{i, j}^{G}$ is the bus voltage which distributed generation $j$ is connected to and $V_{j}^{G, s e t}$ indicates the controlled voltage level.

Formulations on operation of interactive load and battery storage have been discussed in chapter III including formula (2-8).

\subsection{Solution method}

Obviously the formulated multi-period coordinated scheduling model is essentially a typical nonlinear mixed integer programming problem. GAMS could be used to solve this problem.

\section{Case study}

This profit minimization problem is a standard SOCP problem. We used MATLAB on a computer with a Pentium-M $(2.0 \mathrm{GHz})$ processor and 1GB of DDR-RAM and selected CPLEX 12.0 as the solver. 




Fig. 9 The profile of system load

\subsection{Basic data}

\subsubsection{System data}

The proposed model is implemented on the tested REDS (Repository of Distribution Systems) 135-bus distribution system. The test system has been extended from single-period to multi-period, with 1 day as the scheduled duration and $1 \mathrm{~h}$ as basic time interval.

The profile of system load is shown in Fig. 9. The curves with crosses denote active power load; while the ones with triangles denote reactive power load.

\subsection{DER data}

Eight DGs are added, including five GTs, two WTs and one PV; one SD and one CL are added as well.

Table 3 gives the characteristics of the distributed generators. The buses which DGs is connected to are listed in the second column. And the first two DGs are used to simulate wind turbine (W), the last one for photovoltaic (P) and the rest for gas turbine. The power factor of the DGs is set to 0.8 if existed

Table 3 Characteristics of the distributed generators

\begin{tabular}{llllll}
\hline Num & Bus & Type & Power Factor & Voltage & Ramp Rate (kW/15 min) \\
\hline $\mathbf{1}$ & 2 & W & 0.8 & - & - \\
$\mathbf{2}$ & 22 & W & 0.8 & - & - \\
$\mathbf{3}$ & 37 & G & - & 1.05 & 100 \\
$\mathbf{4}$ & 46 & G & 0.8 & - & 100 \\
$\mathbf{5}$ & 62 & G & - & 1.05 & 100 \\
$\mathbf{6}$ & 86 & G & 0.8 & - & 100 \\
$\mathbf{7}$ & 101 & G & - & 1.05 & 100 \\
$\mathbf{8}$ & 121 & P & 0.8 & - & - \\
\hline
\end{tabular}

Table 4 Characteristics of the battery storage

\begin{tabular}{llllll}
\hline Num & Bus & $\begin{array}{l}\text { Initial } \\
\text { electricity/kWh }\end{array}$ & $\begin{array}{l}\text { Maximum } \\
\text { capacity/kWh }\end{array}$ & $\begin{array}{l}\text { Maximum } \\
\text { exchanging } \\
\text { power/kW }\end{array}$ & $\begin{array}{l}\text { Power } \\
\text { exchange } \\
\text { loss }\end{array}$ \\
\hline 1 & 39 & 4000 & 8000 & 500 & 0.05 \\
\hline
\end{tabular}

and the voltage is set to 1.05 , ramp rate is $100 \mathrm{~kW} /$ 15 min.

Table 4 gives the characteristics of the battery storage. There is one battery storage added to the case which is connected to bus 39. Its initial electricity is $4000 \mathrm{kWh}$ and maximum capacity is $8000 \mathrm{kWh}$. Its maximum exchanging power is $500 \mathrm{~kW}$ and power loss rate during charging is 0.05 .

We design one removable load and one shapeable load in the case. Removable load is connected to bus 30, and shapeable load to bus 68 . Their original load shape are shown in Figs. 10 and 11. We design the same load shifting cost curve for them shown in Fig. 12.

\section{Results and discussion}

\subsection{Schedules of the source bus}

The curve with crosses in Fig. 13 shows active power schedules of the source bus; while the one with triangles denotes the total load demand. The trends of the two curves are roughly consistent, with deviations reflecting power outputs from various DERs.

\subsection{Schedules of the DGs}

Figure 14 shows active and reactive power schedules of the DGs. The curves with crosses denote active power schedules; while the ones with triangles denote reactive power schedules.

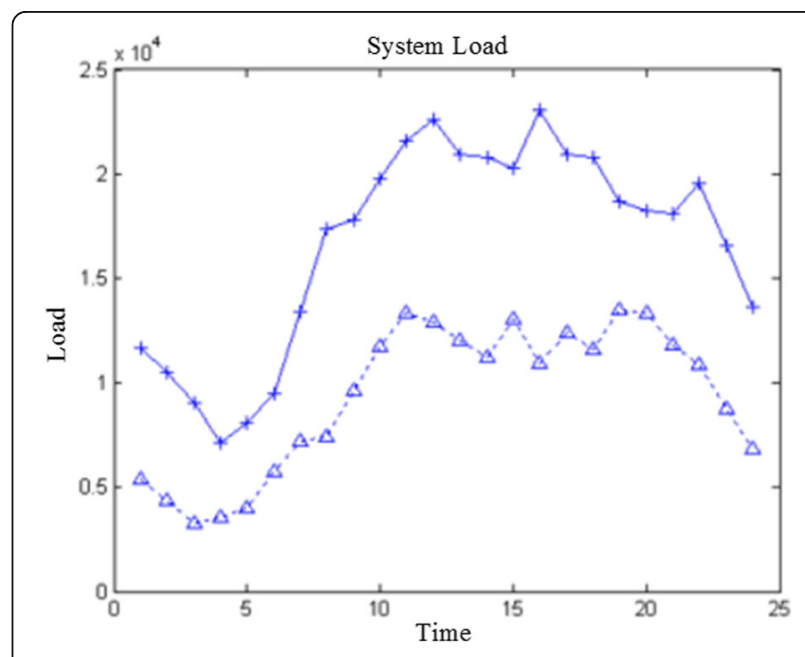

Fig. 10 The profile of original Shapeable Load 


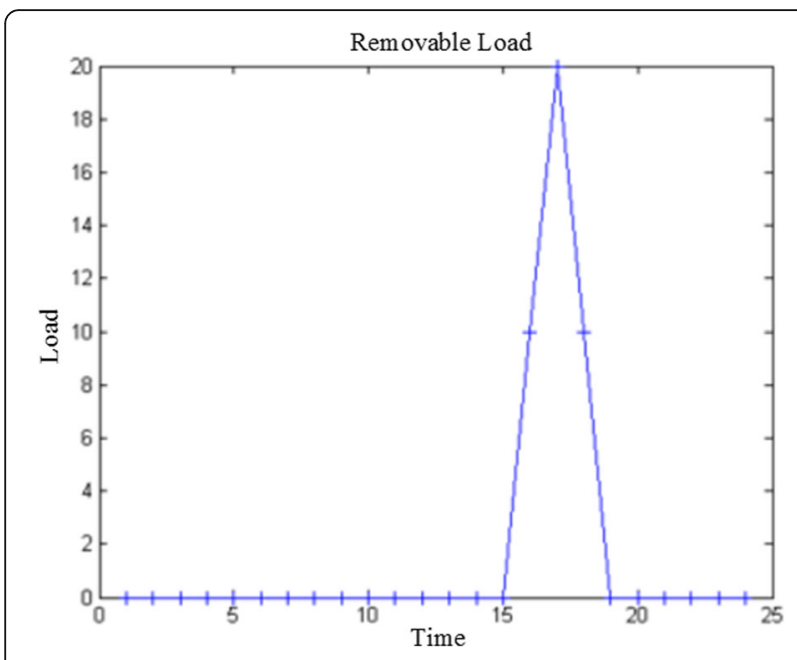

Fig. 11 The Profile of original removable load

No curtailments are observed for WTs and PV, as their capacities are relatively low in this tested system; therefore, the fluctuations could be easily offset by the source bus and the GTs. Reactive power schedules of DG 3, 5 and 7 are appropriately adjusted to keep the bus voltages at the set range, as they adopt CVC control strategy. Therefore, schedules of reactive power are independent of that of active power. However, For DG 4 and 6, as they adopt CPFC strategy, reactive power schedules are proportional to that of active output.

\subsection{Schedules of the storage battery}

Figure 15 shows active power schedule of the SD. Positive values indicate charge state while negative indicate discharge. It could be observed that operation state of the SD is well scheduled, which is in charge state during

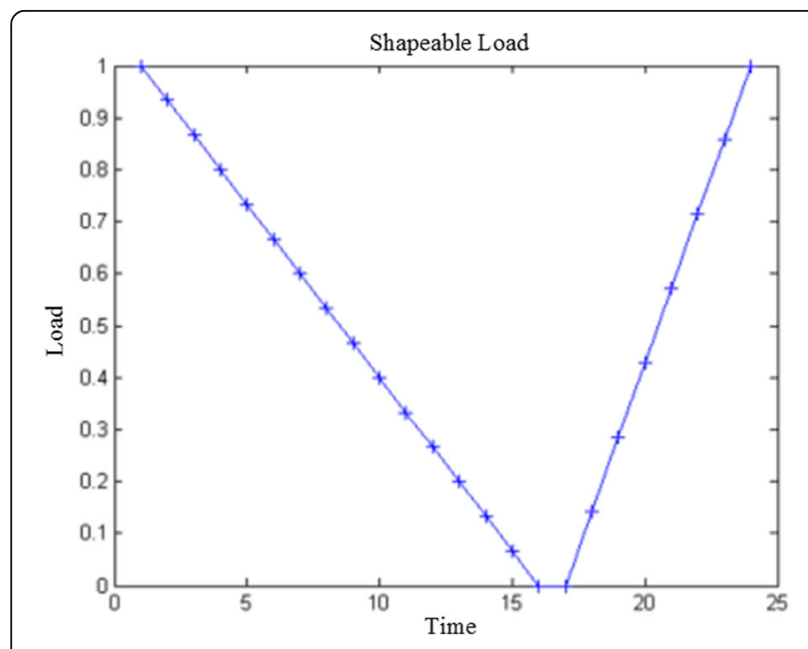

Fig. 12 The profile of load shifting cost

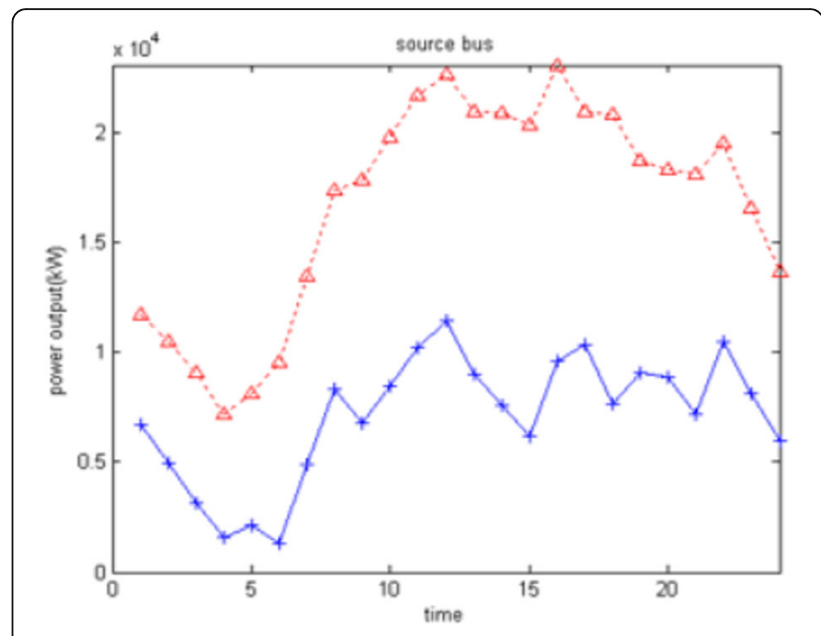

Fig. 13 Active power schedules of the source bus

valley load periods and in discharge state during peak load periods. This schedule could smooth bus load curve and thus might be helpful to reduce overall distribution power losses to some extent.

\subsection{Schedules of the interactive load}

Figure 16 shows active power schedule of the removable load. The curves with crosses denote Original load curve; while the ones with triangles denote load curve after removing. They have the same shapes.

Figure 17 shows active power schedule of the shapeable load. The curves with crosses denote Original load curve; while the ones with triangles denote load curve after removing. Though their have the different shapes, their load stay the same.

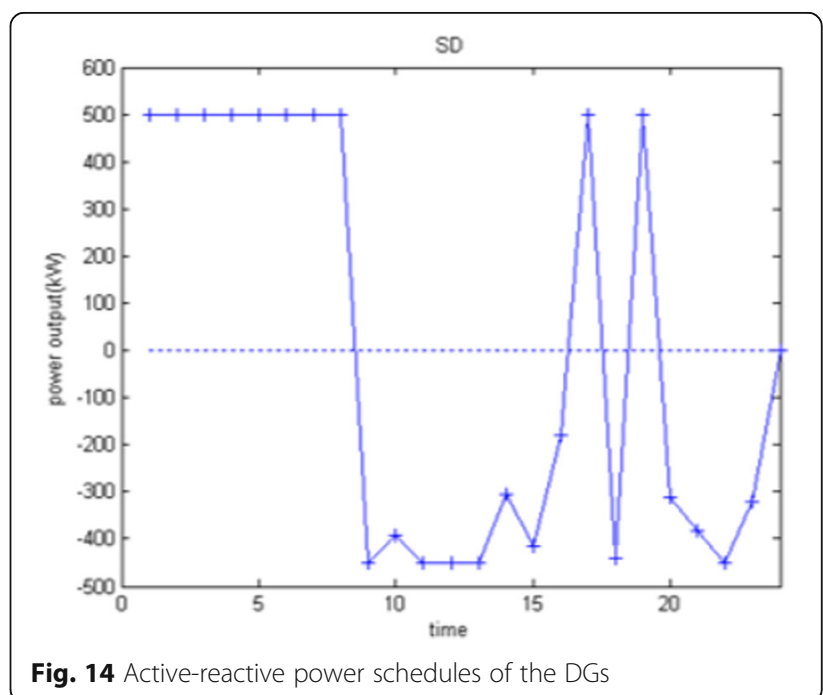




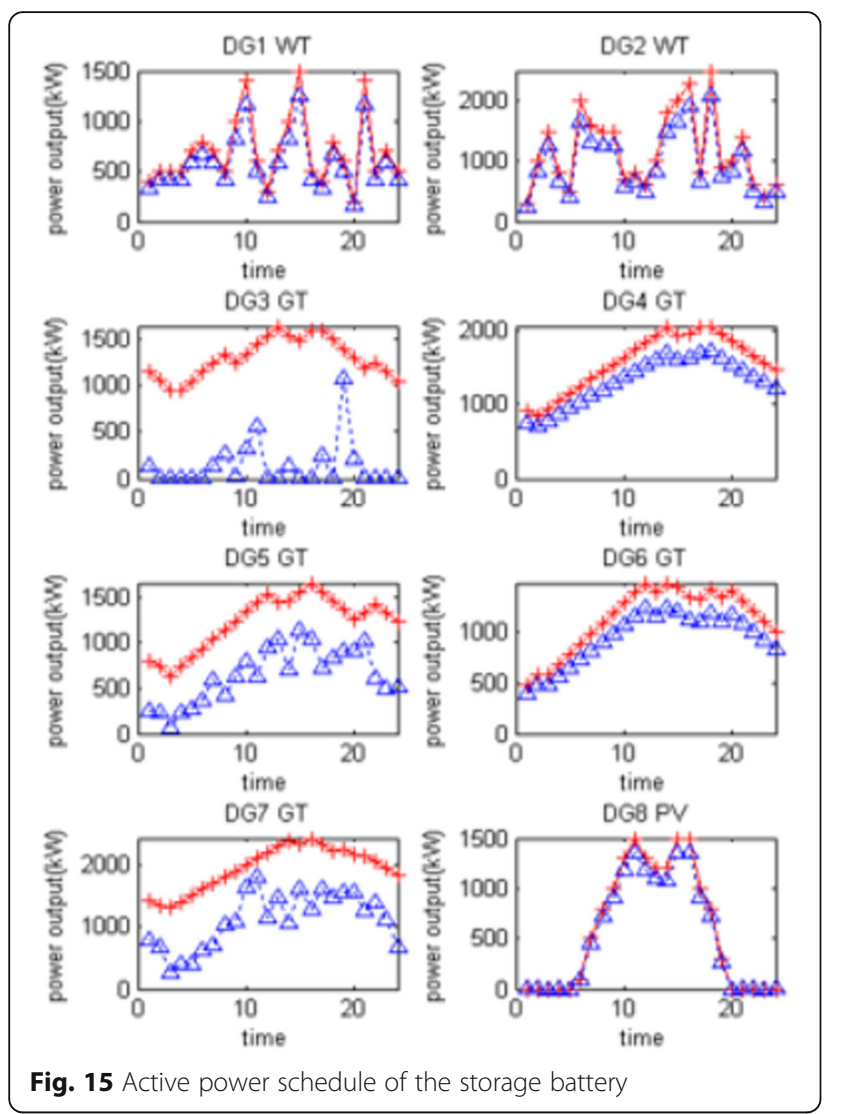

\section{Conclusions}

Implementation of DGs imposes great challenges on traditional distribution system operation. And interactive load and battery storage would reduce their volatility. So scheduling coordinated DG and them will be an important research topic, which imposes remarkable impacts

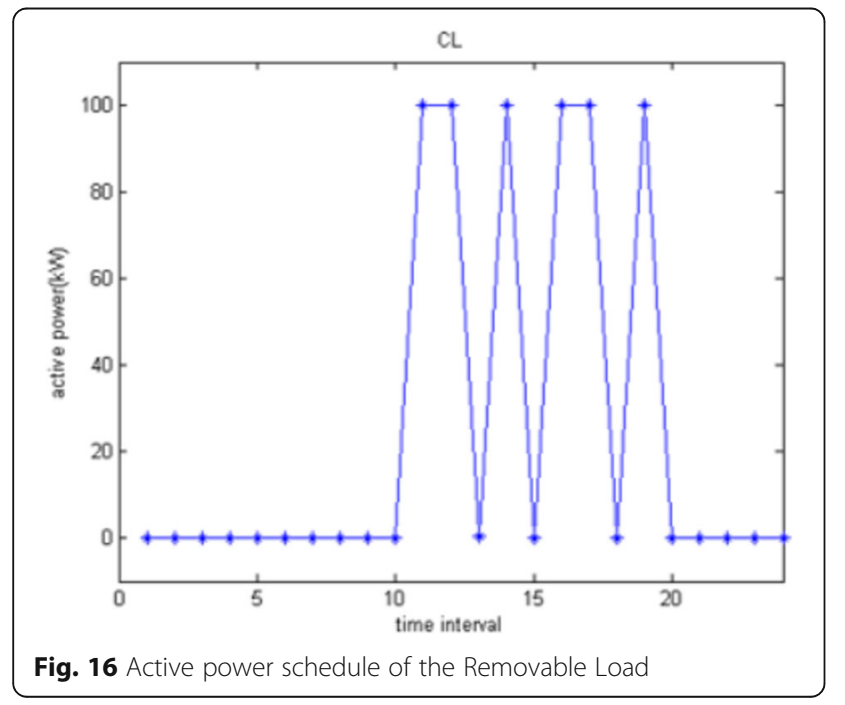

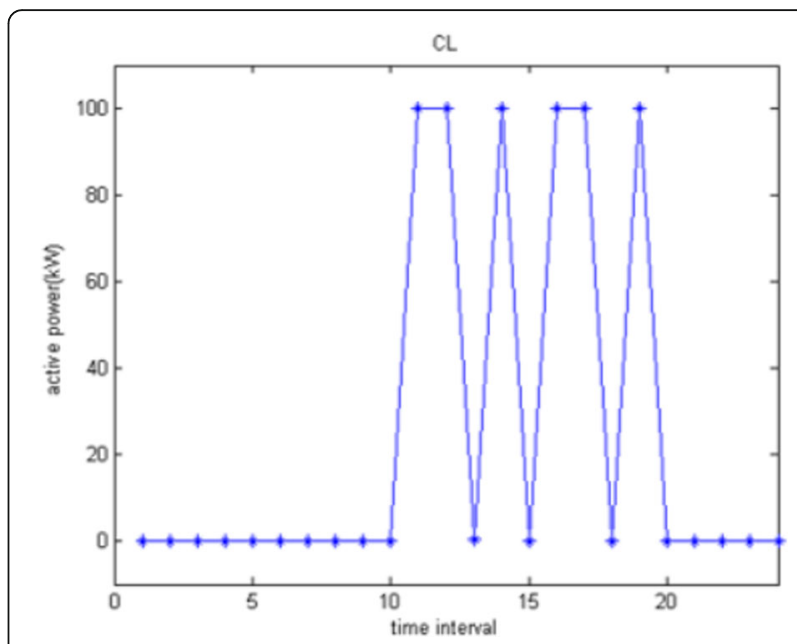

Fig. 17 Active power schedule of the Shapeable Load

on system economics and security. This paper firstly investigates operation characteristics of interactive load and battery storage. Load shifting cost, load shifting positive, load shifting shape and the relation between active and reactive load of interactive load are respectively discussed and formulated. And to battery storage, constraints for energy storage, power exchange, state transition number and operation cost are also formulated in detail. Then, a multi-period coordinated scheduling model is proposed for integrated operation of ADS, with the object of costs minimizing. A tested case is studied, which is based on a 135-bus distribution system with eight DGs, one SD and one CL connected. Solution of the proposed model includes optimal schedules of the DERs, which help to smooth bus load and cut distribution losses in the premise of secure operation constraints.

Authors' contributions

QC: Initiated the research and established models and schedules used for this study, XZ: Cope with establishing the formulation on Operation of Interactive Load and Battery Storage. DG: Manipulated the load data and summarized results in the case study. All authors read and approved the final manuscript.

\section{Competing interests}

The authors declare that they have no competing interests.

Received: 18 April 2017 Accepted: 3 July 2017

Published online: 07 August 2017

\section{References}

1. Pilo, F., Pisano, G., \& Soma, G. G. (2009). "Digital model of a Distribution Management System for the optimal operation of active distribution systems." SmartGrids for Distribution, 2008. IET-CIRED. CIRED Seminar IEEE Xplore, 1-5.

2. Hidalgo, R., Abbey, C., \& Joós, G. (2010). A review of active distribution networks enabling technologies. Power and Energy Society General Meeting IEEE, 1-9. 
3. II-Keun, S., Won-Wook, J., Ju-Yong, K., et al. (2013). Operation schemes of smart distribution networks with distributed energy resources for loss reduction and service restoration. IEEE Transactions on Smart Grid, 4(1), 367-374.

4. Soares, J., et al. (2011). An optimal scheduling problem in distribution networks considering V2G. IEEE, 1-8.

5. Atwa, Y. M., El-Saadany, E. F., Salama, M. M. A., et al. (2010). Optimal renewable resources mix for distribution system energy loss minimization. IEEE Transactions on Power Systems, 25(1), 360-370.

6. Pedrasa, M. A. A., Spooner, T. D., \& Macgill, I. F. (2010). Coordinated scheduling of residential distributed energy resources to optimize smart home energy services. IEEE Transactions on Smart Grid, 1(2), 134-143.

7. Keane, A., \& O'Malley, M. (2007). Optimal utilization of distribution networks for energy harvesting. IEEE Transactions on Power Systems, 22(1), 467-475.

8. Tsikalakis, A. G., \& Hatziargyriou, N. D. (2008). Centralized control for optimizing microgrids operation. IEEE Transactions on Energy Conversion, 23(1), 241-248.

9. Wang, Q., \& Choi, S. S. (2008). The Design of Battery Energy Storage System in a unified power-flow control scheme. IEEE Transactions on Power Delivery, 23(2), 1015-1024.

10. Borghetti, A., Bosetti, M., Grillo, S., et al. (2010). Short-term scheduling and control of active distribution systems with high penetration of renewable resources. IEEE Systems Journal, 4(3), 313-322.

11. Gabash, A., \& Pu, L. (2012). Active-reactive optimal power flow in distribution networks with embedded generation and battery storage. IEEE Transactions on Power Systems, 27(4), 2026-2035.

12. Gabash A, Pu L. Active-reactive optimal power flow for low-voltage networks with photovoltaic distributed generation, 2012.

13. Gabash, A., \& Li, P. (2012). Flexible optimal operation of battery storage Systems for Energy Supply Networks. IEEE Transactions on Power Systems, 99, 1-10.

14. Anderson, M. D., \& Carr, D. S. (1993). Battery energy storage technologies. Proceedings of the IEEE, 81(3), 475-479.

15. Cecati, C., Citro, C., \& Siano, P. (2011). Combined operations of renewable energy systems and responsive demand in a smart grid. IEEE Transactions on Sustainable Energy, 2(4), 468-476.

16. Palensky, P., \& Dietrich, D. (2011). Demand side management: Demand response, intelligent energy systems, and smart loads. IEEE Transactions on Industrial Informatics, 7(3), 381-388.

17. Rahimi, F., \& Ipakchi, A. (2010). Demand response as a market resource under the smart grid paradigm. IEEE Transactions on Smart Grid, 1(1), 82-88.

18. Ruiz, N., Cobelo, l., \& Oyarzabal, J. (2009). A direct load control model for virtual power plant management. IEEE Transactions on Power Systems, 24(2), 959-966.

\section{Submit your manuscript to a SpringerOpen ${ }^{\circ}$ journal and benefit from:}

- Convenient online submission

- Rigorous peer review

- Open access: articles freely available online

- High visibility within the field

- Retaining the copyright to your article 